\title{
PHOTOLUMINESCENCE OF Co-DOPED ZnCdSe AND ZnSSe ALLOYS
}

\author{
T.P. SURKOVA \\ Institute of Metal Physics, Ural Division of Russian Academy of Sciences \\ 620219 Ekaterinburg GSP-170, Russia
}

H. Born, P. Thurian, A. Hoffmann, W. Busse, H.-E. Gumlich, I. BROSER

Technical University Berlin, Institute of Solid State Physics, 10623 Berlin, Germany

AND W. GIRIAT

IVIC, Centro de Fisica, Apto 1827, Caracas 1010A, Venezuela

Photoluminescence measurements have been carried out for $\mathrm{Zn}_{1-x} \mathrm{Cd}_{x} \mathrm{Se}$ :Co and $\mathrm{ZnS}_{x} \mathrm{Se}_{1-x}$ :Co mixed crystals. Changes of recombination channels are observed in mixed crystals as compared to the emission of host binary compounds. Character of changes is slightly different for the alloys with cation and anion substitution. Photoluminescence kinetics of the $L$-line and of two other $\mathrm{Co}^{2+}$ intra-shell emission bands was measured to determine radiative decay rates.

PACS numbers: 71.55.Gs, 78.20.Wc

Transition metal impurities are well known as optically active centres in semiconductors. They have been the subject of manifold experimental and theoretical investigations for binary II-VI and III-V compounds. Their properties in ternary mixed crystals are, however, less understood. In this paper we present the first results of photoluminescence (PL) study of cobalt doped $\mathrm{Zn}_{1-x} \mathrm{Cd}_{x} \mathrm{Se}$ and $\mathrm{ZnS}_{x} \mathrm{Se}_{1-x}$ alloys.

PL spectra were measured at low temperature for the ternary alloys of $\mathrm{ZnCdSe}$ and $\mathrm{ZnSSe}$ with the wide composition range of $\mathrm{Cd}(x=0 \div 0.5)$ and $\mathrm{S}(x=0 \div 1.0)$. All the samples studied were grown by a chemical transport method and were doped with Co to the level of $10^{18} \mathrm{~cm}^{-3}$. The PL excitation was provided with $441.6 \mathrm{~nm} \mathrm{He}-\mathrm{Cd}$ laser line, and PL spectra were recorded at $5 \mathrm{~K}$ with a SPEX 1401 spectrometer working in the spectral range of $0.7-2.8 \mathrm{eV}$.

The results of PL investigations are presented in Figs. 1 and 2. The PL spectra of ternary crystals with relatively small $\mathrm{Cd}$ and $\mathrm{S}$ content are dominated by the so-called $L$-line emission, which for the binary $\mathrm{ZnSe:Co}$ crystals was related to the 


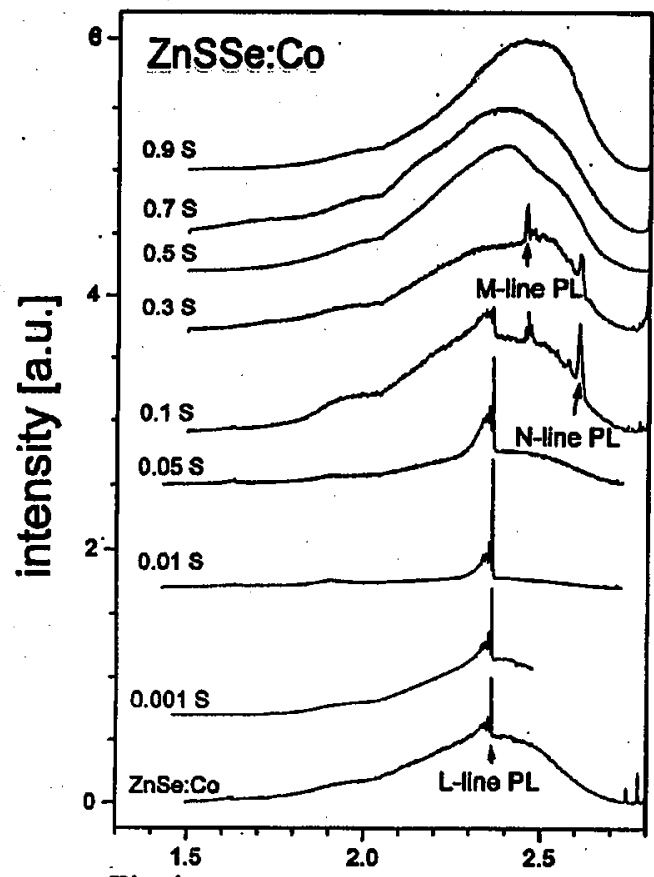

Fig.1

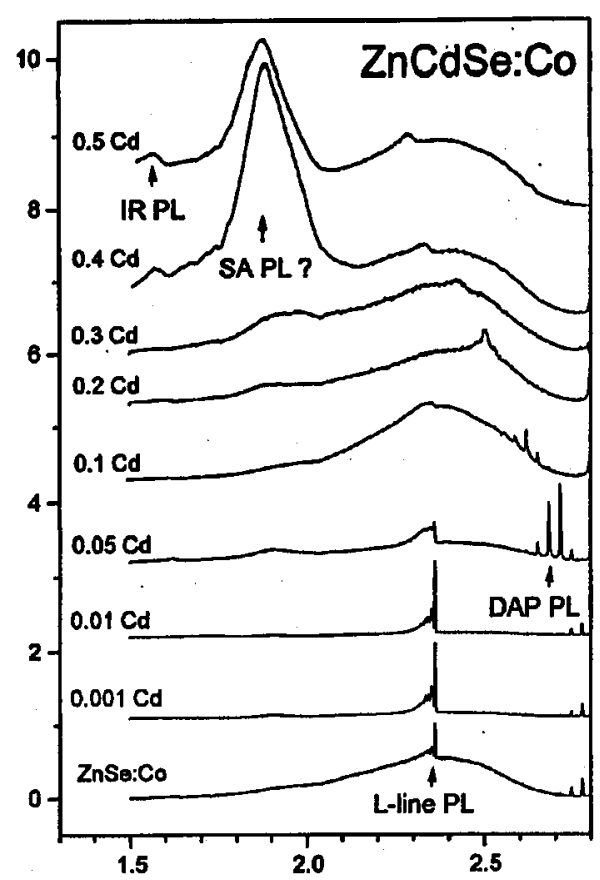

Fig.2 Energy [eV]

Fig. 1. PL spectra of $\mathrm{ZnSe:Co}$ and $\mathrm{ZnS}_{x} \mathrm{Se}_{1-x}$ :Co mixed crystals $(0<x<0.9)$ recorded at $5 \mathrm{~K}$ under excitation with $\mathrm{He}-\mathrm{Cd}$ laser $(441.6 \mathrm{~nm})$.

Fig. 2. PL spectra of $\mathrm{ZnSe}$ :Co and $\mathrm{Zn}_{1-x} \mathrm{Cd}_{x}$ Se:Co mixed crystals $(0<x<0.5)$ recorded at $5 \mathrm{~K}$ under excitation with $\mathrm{He}-\mathrm{Cd}$ laser $(441.6 \mathrm{~nm})$.

doublet-quartet intra-shell $d-d$ PL transition of $\mathrm{Co}^{2+}$ from unidentified doublet state to the ground ${ }^{4} A_{2}(F)$ state $[1,2]$. For higher $\mathrm{Cd}$ and $\mathrm{S}$ fractions the character of PL changes is different for $\mathrm{Zn}_{1-x} \mathrm{Cd}_{x}$ Se:Co and $\mathrm{ZnS}_{x}$ Se $\mathrm{e}_{1-x}$ :Co alloys. For $\mathrm{ZnS}_{x} \mathrm{Se}_{1-x}$ :Co crystals $(0.3>x \geq 0.1)$ besides the $L$-line two additional PL bands with a fine structure appear; these are analogies of the $M$ - and $N$-lines observed in absorption spectra of binary ZnSe:Co crystals [1], but not in PL spectra [3]. These two transitions involve two unidentified doublet excited states of $\mathrm{Co}^{2+}$, likely of the same origin as the $L$-line related state [3], located very close to the conduction band edge of ZnSe. For ternary ZnSSe alloys these two states separate from the conduction band edge and PL emission from these states can be observed. This effect, rather than a change of a local symmetry in the alloys, results in $M$ - and $N$-line PL.

For $\mathrm{Zn}_{1-x} \mathrm{Cd}_{x}$ Se:Co alloys $L$-line and its phonon replica are observed till $x=0.05$ (see Fig. 2), i.e., the effect of cation substitution on PL alloy broadening is more pronounced. Figure 2 shows also that two additional PL spectra are observed. The first emission, with a fine structure, is observed near the band gap energy. This PL is probably due to a shallow donor-shallow acceptor pair (DAP PL in Fig. 2) 
recombination and shifts down in energy with increasing $\mathrm{Cd}$ fraction following the changes of the energy gap. The second broad and intensive PL dominates in alloys with a large $\mathrm{Cd}$ content. The nature of this broad band is unknown, but likely it is an analogue of a self-activated PL (SA PL) often observed for the wide band gap II-VI compounds. Besides the strong PL in the visible region of emission, two broad and weak infrared (IR PL in Fig. 2) emissions are observed in the spectral range of 1.5-1.7 eV. These bands were also observed for the ZnSSe:Co alloys but were weaker and are not seen in Fig. 1.

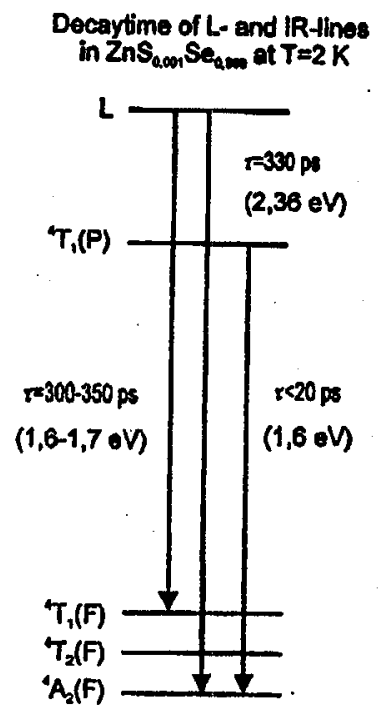

Fig. 3. Model of energy level scheme for radiative recombination transitions of $\mathrm{Co}^{2+}$ ion in $\mathrm{ZnSe}$ and its alloys with $\mathrm{CdSe}$ and $\mathrm{ZnS}$. PL decay times measured for $\mathrm{ZnS}_{0.001} \mathrm{Se}_{0.999}$ :Co are given.

We have studied PL kinetics to determine identity of the observed PL bands. The PL decay time of the $L$-line emissions depends strongly on the Cd and S composition. Whereas for the pure $\mathrm{ZnSe:Co}$ this decay time is about $2 \mathrm{~ns}$, it rapidly decreases to about 330 ps for $\mathrm{ZnS}_{0.001} \mathrm{Se}_{0.999}$ :Co (Fig. 3). Such rapid decay suggests very enhanced nonradiative recombination transitions. The PL kinetics studies (PL decay times measured are given in Fig. 3) allowed a clear distinction between corresponding spin-allowed ${ }^{4} T_{1}(P) \rightarrow{ }^{4} A_{2}(F)$ transition and spin-forbidden transitions from the excited doublet state ( $L$ level in Fig. 3) to either the ${ }^{4} T_{1}(F)$ or to the ground ${ }^{4} A_{2}(F)$ state. We conclude that infrared PL at about $1.6 \mathrm{eV}$ consists of two overlapping bands with different spin selection rules for PL recombination. Of these two bands one starts from the same excited state as the $L$-line PL, as is shown in Fig. 3.

Concluding, recombination channels in alloys of $\mathrm{Zn}_{1-x} \mathrm{Cd}_{x} \mathrm{Se}$ :Co and $\mathrm{ZnS}_{x} \mathrm{Se}_{1-x}$ :Co were studied. We report different PL changes for alloys with cation and anion substitution. However, the character of the changes depends mainly on 
a relative position of the excited $\mathrm{Co}^{2+}$ levels versus edges of conduction band in alloys than on e.g. alloy broadening. From PL kinetics measurements we resolve also overlapping PL bands in the infrared region of the emission.

This work has been partly supported by the RFBR (grant N 96-02-16496) and INTAS (grant N 93-3657). One of us (T.P. Surkova) was supported by a scholarship of the D.A.A.D.

\section{References}

[1] D.J. Robbins, P.J. Dean, J.L. Glasper, S.G. Bishop, Solid. State Commun. 36, 61 (1980).

[2] J. Dreyhsig, H.-E. Gumlich, J.W. Allen, Phys. Rev. B 48, 15002 (1993).

[3] J. Dreyhsig, Ph.D. Thesis, TU, Berlin 1994. 